\title{
Prognostic value of soluble ST2 postaortic valve replacement: a meta-analysis
}

\author{
Gary Tse, ${ }^{1,2}$ Christina Ip ${ }^{1,2}$ King Sum Luk, ${ }^{1,2}$ Mengqi Gong, ${ }^{3}$ Yan Yee Ting, ${ }^{1,2}$ \\ Ishan Lakhani, ${ }^{1,2}$ George Bazoukis, ${ }^{4}$ Guangping Li, ${ }^{3}$ Konstantinos P Letsas, ${ }^{4}$ \\ Mei Dong, ${ }^{5}$ Tong Liu, ${ }^{3}$ Martin C S Wong ${ }^{6}$
}

\begin{abstract}
- Additional material is published online only. To view please visit the journal online (http://dx.doi.org/10.1136/ heartasia-2017-010980).

'Department of Medicine and Therapeutics, Faculty of Medicine, Chinese University of Hong Kong, Hong Kong, China ${ }^{2}$ Faculty of Medicine, Li Ka Shing Institute of Health Sciences, Chinese University of Hong Kong, Hong Kong, China ${ }^{3}$ Tianjin Key Laboratory of Ionic-Molecular Function of Cardiovascular Disease, Department of Cardiology, Tianjin Institute of Cardiology, Second Hospital of Tianjin Medical University, Tianjin, China

${ }^{4}$ Laboratory of Cardiac Electrophysiology, Second Department of Cardiology, 'Evangelismos' General Hospital of Athens, Athens, Greece ${ }^{5}$ Department of Cardiology, Yantai Yuhuangding Hospital Affiliated to Qingdao University, Yantai, China

${ }^{6} \mathrm{JC}$ School of Public Health and Primary Care, The Chinese University of Hong Kong, Hong Kong, China
\end{abstract}

\section{Correspondence to} Dr Gary Tse, Department of Medicine and Therapeutics, Faculty of Medicine, Chinese University of Hong Kong, Hong Kong, China; tseg@cuhk.edu.hk and Professor Martin C SWong, JC School of Public Health and Primary Care The Chinese University of Hong Kong Hong Kong China ; wong_martin@ cuhk.edu.hk

Received 14 November 2017 Revised 19 February 2018 Accepted 21 February 2018

Check for updates

To cite: Tse $\mathrm{G}, \mathrm{Ip} C$ Luk KS, et al. Heart Asia 2018;10:1-4. doi:10.1136/ heartasia-2017-010980

\section{ABSTRACT \\ Objectives Soluble suppression of tumorigenicity 2} (sST2) is a member of the interleukin-1 receptor family and a modulator of hypertrophic and fibrotic responses. Its prognostic value for patients undergoing aortic valve replacement (AVR) has been examined in prospective studies but to date, there has been no systematic evaluation or meta-analysis on this issue.

Methods PubMed and Embase were searched until 1 October 2017 for studies that evaluated the relationship between SST2 levels and mortality after AVR.

Results A total of 18 and 37 entries were retrieved from both databases, from which four studies were included in the final meta-analysis. In a total of 1154 subjects ( $50 \%$ male, mean age $80 \pm 10$ years old, mean follow-up 14 months), elevated SST2 levels were significantly associated with a $44 \%$ increase in the risk of all-cause mortality (HR $1.44,95 \% \mathrm{Cl} 1.30$ to 1.60 , $p<0.0001 ;\left.\right|^{2}: 44 \%$ ).

Conclusions SST2 significantly predicts all-cause mortality in patients who have undergone AVR, but this conclusion is limited by the small number of patients. Larger prospective studies are required to better elucidate its value for risk stratification in this patient population.

\section{INTRODUCTION}

Aortic stenosis (AS) is a progressive disease that can lead to left ventricular hypertrophy and ventricular fibrosis ${ }^{1}$ with a prevalence of around $3.4 \%$ of individuals above the age of $75 .^{2}$ Traditionally, aortic valve replacement (AVR) is performed by conventional surgery, but transcatheter aortic valve implantation (TAVI) is increasingly used for those who have severe, symptomatic AS. ${ }^{3}$ However, not all patients benefit from AVR, such as those who are frail. ${ }^{4} \mathrm{~A}$ number of risk scores, such as the European System for Cardiac Operative Risk Evaluation II, Society of Thoracic Surgeons score, or German Aortic Valve score, have been devised for risk stratification but these generally have poor predictive valves in patients with AS undergoing AVR. ${ }^{5}$ Imaging techniques such as MRI are also helpful. ${ }^{6}$ More recently, there has been interest in using different serum biomarkers for this purpose. Of these, soluble suppression of tumorigenicity 2 (sST2), a member of the interleukin (IL)-1 receptor family, has emerged to be a promising marker of myocardial stress. ${ }^{7}$ Thus, it has been shown to be predictive of adverse clinical outcomes in different cardiovascular diseases, such as stable coronary artery disease, ${ }^{8}$ acute coronary syndrome, ${ }^{9}$ and acute ${ }^{10}$ as well as chronic heart failure. ${ }^{11}$
In the context of AS, some studies have demonstrated significant prognostic value of sST2 in patients undergoing AVR. ${ }^{12}$ However, although Stundl and colleagues reported that this biomarker was a significant predictor of mortality in a study cohort, it did not do so in a separate independent validation cohort. ${ }^{13}$ Therefore, we conducted this systematic review and meta-analysis to evaluate the relationship between serum sST2 level and mortality outcomes in this patient population.

\section{METHODS}

\section{Search strategy, inclusion and exclusion criteria}

This systematic review and meta-analysis was conducted using the Preferred Reporting Items for Systematic Reviews and Meta-Analyses checklist. We searched two databases, PubMed and Embase, for studies that examined the association between sST2 levels and AVR using the following terms: ((Soluble suppression of tumorigenicity-2 OR sST2) and aortic). The search period was from the beginning of the databases up to 1 October 2017, with no language restrictions using the following inclusion criteria: (1) the design was a case-control, prospective or retrospective cohort study in human subjects; (2) sST2 values were provided and related to disease severity or mortality in patients who have undergone AVR.

The Newcastle-Ottawa Quality Assessment Scale (NOS) was used for assessing the quality of the included studies (online supplementary table 1), giving a score of $0-9$ for each study: $<5$ indicated poor quality, 5-7 indicated fair quality, and $>8$ indicated good quality.

\section{Data extraction and statistical analysis}

The extracted data were input into Microsoft Excel software. Full articles of all entries were retrieved and assessed to determine whether all of the inclusion criteria were met. In this study, the extracted data elements consisted of: (1) publication details: surname of the first author, publication year; (2) the study design; (3) follow-up period; (4) the NOS quality score; and (5) the characteristics of the population including sample size, sex, age and cut-off for sST2. Two reviewers (GT and $\mathrm{CI}$ ) independently reviewed each included study and disagreements were resolved by consulting a third reviewer (TL). For the relationship between sST2 and mortality, multivariate adjusted HRs with 95\% CI were extracted and analysed for each study. Heterogeneity across studies was determined 


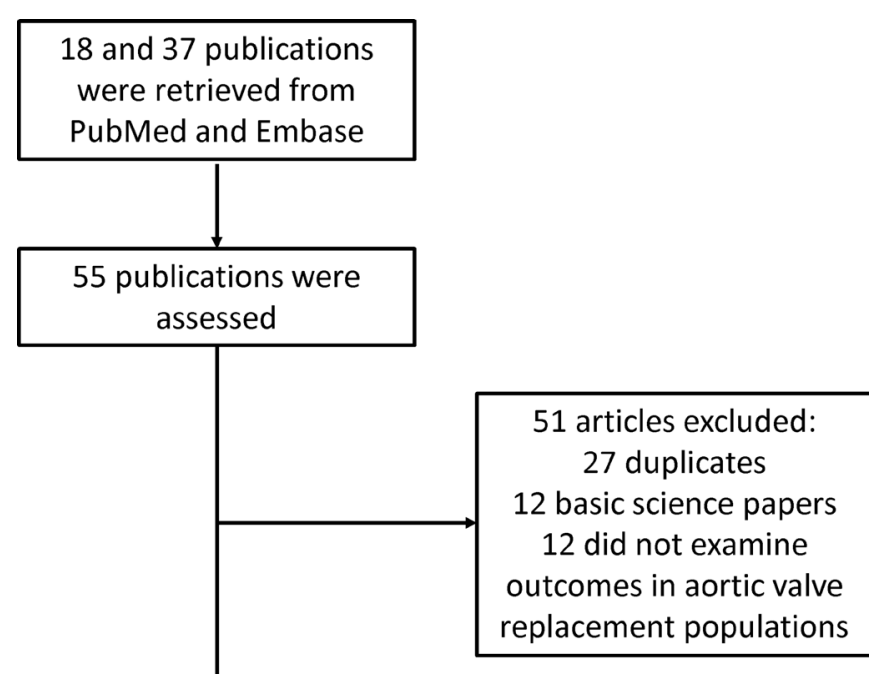

4 publications included in

the final meta-analysis

Figure 1 Flow chart of the study selection process.

using Cochran's $\mathrm{Q}$ value and the $\mathrm{I}^{2}$ statistic. $\mathrm{I}^{2}>50 \%$ was considered to reflect significant statistical heterogeneity. If $\mathrm{I}^{2}$ was less than $50 \%$, the fixed effects model was used, otherwise the random effects model was used. To identify the origin of the heterogeneity, sensitivity analysis using the leave-one-out method was performed. Publication bias and asymmetry were assessed using the Begg and Mazumdar rank correlation test and Egger's test, respectively.

\section{RESULTS}

The search strategy of this systematic review and meta-analysis is shown in figure 1. A total of 18 and 37 entries were retrieved from PubMed and Embase, respectively. After excluding duplicate studies or duplicate study populations $(n=27)$, basic science studies $(n=12)$ and studies that did not investigate outcomes following AVR ( $n=12)$, four studies were included in the final meta-analysis. ${ }^{12-15}$ The baseline characteristics of these studies are listed in table 1 . Three were prospective studies ${ }^{12-14}$ and one was a retrospective study. ${ }^{15}$ Three studies examined outcomes after TAVI, whereas one study examined outcomes after AVR using either TAVI or conventional surgery. The present meta-analysis included 1154 subjects $(50 \%$ male, mean age $80 \pm 10$ years old, mean follow-up 14 months). Four studies evaluated the value of sST2 in predicting all-cause mortality, all of which demonstrated significant associations. However, in the Stundl et al's study, ${ }^{13}$ sST2 was not a significant predictor of mortality in the validation cohort. Nevertheless, our meta-analysis shows that elevated sST2 levels were significantly associated with a 44\% increase in the risk of all-cause mortality (HR 1.44, $95 \%$ CI 1.30 to $1.60, \mathrm{p}<0.0001$; figure 2 ). $\mathrm{I}^{2}$ was $44 \%$, indicating the presence of moderate heterogeneity. A funnel plot of SE against the logarithm of HR is shown in figure 3. Begg and Mazumdar rank correlation test suggested no significant publication bias (Kendal's tau value 0.4, $\mathrm{p}>0.05$ ). Egger's test demonstrated no significant asymmetry (intercept $1.5, \mathrm{t}$ value 1.6; $\mathrm{p}>0.05$ ). Sensitivity analysis excluding one study at a time did not significantly affect the pooled HR (figure 4). Excluding the Lindman et al's study ${ }^{14}$ that examined patients undergoing both TAVI and conventional surgery, heterogeneity was reduced to $36 \%$. When follow-up duration was analysed separately, including studies that examined 12-month mortality ${ }^{13} 15$ reduced heterogeneity to $20 \%$.

\section{DISCUSSION}

The main findings of this systematic review and meta-analysis are that high levels of sST2 were associated with a $44 \%$ increase in all-cause mortality in patients with AS undergoing AVR.

Over the past decade or so, several biomarkers have emerged that have been shown to provide further prognostic value in addition to traditional clinical risk scores. For example, the biomarker B-type natriuretic peptide (BNP) is produced in response to pressure overload, ${ }^{16}$ whereas sST2 is released during chronic inflammation. ${ }^{17}$ The biomarker sST2 is a member of the IL-1 receptor family with both transmembrane (ST2L) and soluble (SST2) isoforms. ${ }^{18}$ The latter acts as a decoy receptor for IL-33, preventing the interaction between IL-33 and ST2L. In AS, increased diastolic load causes sST2 release from endothelial cells and cardiomyocytes, ${ }^{19}$ thereby leading to a proinflammatory milieu. ${ }^{20}$ sST2 has demonstrated a prognostic value in the context of pulmonary hypertension. ${ }^{21}$ Our systematic review and meta-analysis demonstrates its value in predicting all-cause mortality for patients undergoing AVR. The cut-off value of sST2 used in the included studies ranged from 10 to $49 \mathrm{ng} / \mathrm{mL}$ with a mean value of $31 \mathrm{ng} / \mathrm{mL}$. Interestingly, sST2 was shown to outperform $\mathrm{BNP},{ }^{12}$ possibly due to $\mathrm{SST} 2$ being less prone to haemodynamic fluctuations, in contrast to natriuretic peptides that are released with cardiac filling as well as increased wall stress. Moreover, since sST2 release is dependent on chronic

Table 1 Characteristics of the four studies included in this meta-analysis

\begin{tabular}{|c|c|c|c|c|c|c|c|c|}
\hline $\begin{array}{l}\text { First author/ } \\
\text { year }\end{array}$ & Population & $\begin{array}{l}\text { Sample } \\
\text { size }(n)\end{array}$ & $\begin{array}{l}\text { Age } \\
\text { (years) }\end{array}$ & Male, $\mathrm{n}$ & $\begin{array}{l}\text { Follow-up } \\
\text { (months) }\end{array}$ & Cut-off (ng/mL) & Variables in multivariate model & Ref \\
\hline Schmid, 2017 & TAVI & 74 & 83 & 26 & 31 & 49 & STS score, RA area & 12 \\
\hline Stundl, 2017 & TAVI & 461 & 81 & 234 & 12 & $\begin{array}{l}29 \text { (development cohort); } \\
33 \text { (validation cohort) }\end{array}$ & $\begin{array}{l}\text { LES, EuroSCORE II (ES II), STS-PROM, CRF, previous } \\
\text { cardiac surgery, pulmonary hypertension, New York Heart } \\
\text { Association class IV, NT-proBNP, LVEF }\end{array}$ & 13 \\
\hline Wernly, 2017 & TAVI & 274 & 80 & 125 & 12 & 10 & $\begin{array}{l}\text { STS score, EuroSCORE, CRP, LVEDD, IDDM, LVEF, major } \\
\text { vascular complication, TnT-I, SPAP, mean pressure gradient, } \\
6 \text { min walk test }\end{array}$ & 15 \\
\hline Lindman, 2015 & $\begin{array}{l}\text { AVR (TAVI } \\
\text { and surgical) }\end{array}$ & 345 & 78 & 194 & 23 & 33 & $\begin{array}{l}\text { Glomerular filtration rate, sex, diabetes mellitus, obstructive } \\
\text { lung disease, New York Heart Association functional class } \\
\text { (III/IV vs I/III), aortic mean gradient }\end{array}$ & 14 \\
\hline
\end{tabular}

AVR, aortic valve replacement; CRF, chronic renal failure; CRP, C-reactive protein; ES II, European System for Cardiac Operative Risk Evaluation II; IDDM, insulin-dependent diabetes mellitus; LES, logistic EuroSCORE; LVEDD, left ventricular end-diastolic volume; LVEF, left ventricular ejection fraction; NT-proBNP, N-terminal pro B-type natriuretic peptide; RA, right atrium; sPAP, systolic pulmonary arterial pressure; STS, Society of Thoracic Surgeons; STS-PROM, STS predicted risk of mortality; TAVI, transcatheter aortic valve implantation; TnT, troponin 
High sST2 levels and risk of all-cause mortality

\begin{tabular}{lccccrr} 
Study name & \multicolumn{5}{c}{ Statistics for each study } \\
\cline { 2 - 6 } & $\begin{array}{c}\text { Hazard } \\
\text { ratio }\end{array}$ & $\begin{array}{c}\text { Lower } \\
\text { limit }\end{array}$ & $\begin{array}{c}\text { Upper } \\
\text { limit }\end{array}$ & Z-Value & p-Value \\
Schmid 2017 & 2.920 & 1.100 & 7.751 & 2.151 & 0.031 \\
Stundl 2017 & 1.295 & 1.121 & 1.497 & 3.503 & 0.000 \\
Stundl 2017 (validation cohort) & 1.410 & 0.814 & 2.442 & 1.226 & 0.220 \\
Wemly 2017 & 1.541 & 1.314 & 1.807 & 5.321 & 0.000 \\
Lindman 2015 & 2.040 & 1.309 & 3.178 & 3.151 & 0.002 \\
& 1.441 & 1.301 & 1.595 & 7.035 & 0.000
\end{tabular}

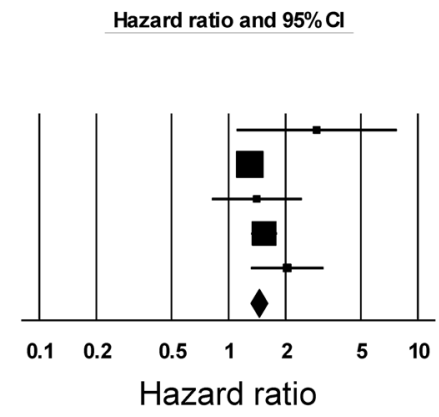

Lower risk Higher risk

Figure 2 HRs for soluble suppression of tumorigenicity 2 (SST2) and all-cause mortality in patients undergoing aortic valve replacement.

Funnel Plot of Standard Error by Log hazard ratio

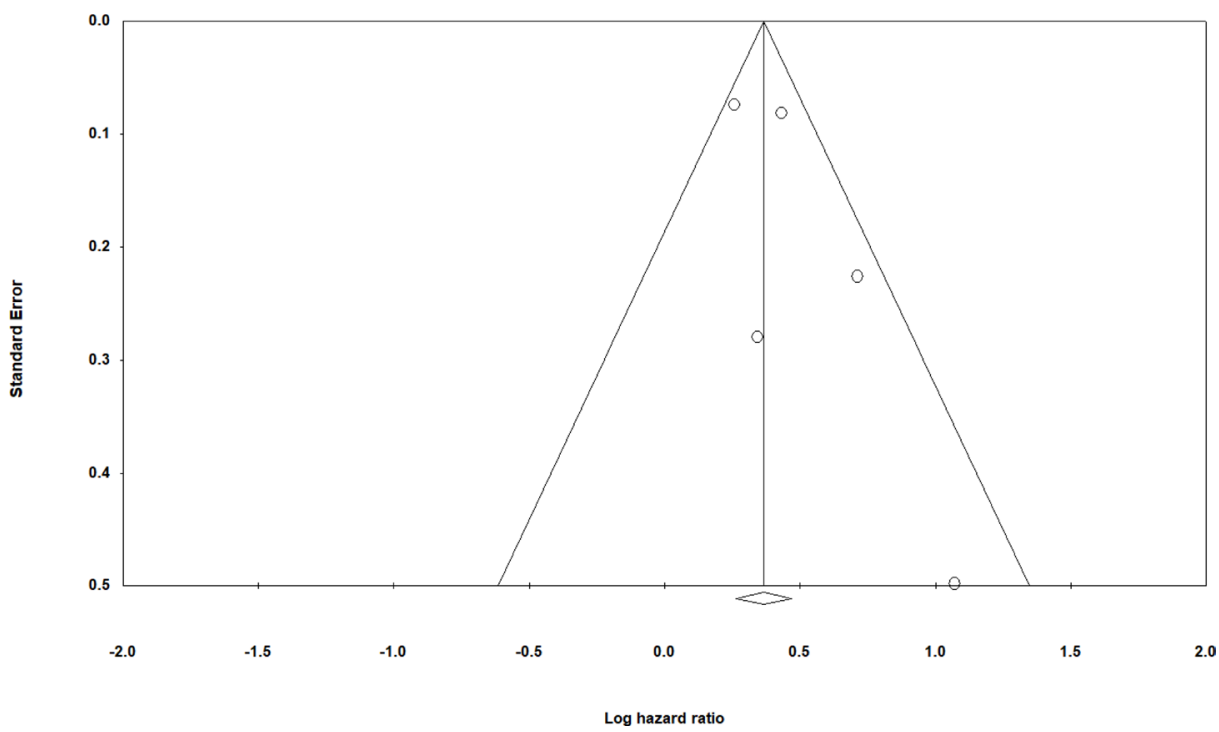

Figure 3 Funnel plot of SE against the logarithm of the HR.

inflammation, this may adversely affect outcomes in patients undergoing AVR.

\section{Strengths and limitations}

There are many strengths of this study. First, HRs from multivariate analysis were available from all of the included studies, reducing the likelihood of confounders. Second, heterogeneity was less than $50 \%$, meaning that the fixed effects model was appropriate for pooling of the HRs. Nevertheless, several limitations should be acknowledged. For example, all but one of the included studies had a prospective design, meaning that certain types such as recall bias may be present. Some degree of heterogeneity was observed; this was likely due to a combination of the different methods of AVR (TAVI only vs TAVI or conventional surgery), follow-up duration, different cut-off values used and different variables included in multivariate analysis. Moreover, the use of different cut-off values may lead to different HRs. We had aimed to perform a subgroup analysis
Study name

Schmid 2017

Stundl 2017

Stundl 2017 (validation cohort)

Wemly 2017

\begin{tabular}{|c|c|c|c|c|}
\hline \multicolumn{5}{|c|}{ Statistics with study removed } \\
\hline oint & $\begin{array}{c}\text { Lower } \\
\text { limit }\end{array}$ & $\begin{array}{c}\text { Upper } \\
\text { limit }\end{array}$ & Z-Value & p-Value \\
\hline 401 & 1.261 & 1.557 & 6.291 & \\
\hline 554 & 1.336 & 1.808 & 5.719 & \\
\hline 413 & 1.270 & 1.572 & 6.368 & \\
\hline 323 & 1.152 & 1.520 & 3.968 & \\
\hline 413 & 1.273 & 1.569 & 6.485 & \\
\hline
\end{tabular}

Figure 4 Sensitivity analysis using the leave-one-out method.

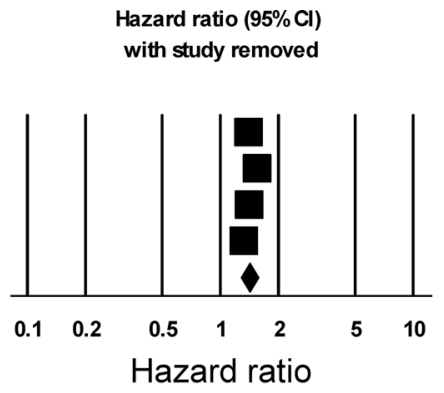

Lower risk Higher risk 
based on the type of AVR (TAVI vs aortic valve surgery) but only one study included patients undergoing surgery. It was therefore not possible to conduct this subgroup analysis. Nevertheless, sensitivity analysis by excluding the Lindeman et al's study that included both TAVI and aortic valve surgery patients ${ }^{14}$ did not significantly alter the pooled HR. Finally, the sample size of the included cohort was small.

sST2 has been used for predicting prognosis in a number of cardiovascular conditions and may provide incremental value for risk stratification. As the study population and inclusion criteria were prespecified to include patients undergoing AVR only, outcomes in these conditions were not examined. Further meta-analyses are needed to confirm whether sST2 also provides a prognostic value in these conditions.

\section{CONCLUSION}

sST2 significantly predicts all-cause mortality in patients who have undergone AVR, but this conclusion is limited by the small number of patients. Larger prospective studies are required to better elucidate its value for risk stratification in this patient population.

\section{Key messages}

What is already known about this subject?

- Soluble suppression of tumorigenicity 2 (SST2) is associated with increased cardiovascular risk. Its prognostic value in patients undergoing aortic valve replacement has been examined in previous studies but to date, there has been no systematic evaluation of this biomarker.

\section{What does this study add?}

- SST2 significantly predicts all-cause mortality in patients who have undergone aortic valve replacement.

How might this impact on clinical practice?

- SST2 is a useful biomarker for predicting mortality in aortic valve replacement. It provides additional value for risk stratification but larger prospective studies are needed to confirm the relationship and its value as a biomarker.

Acknowledgements GT thanks the Croucher Foundation of Hong Kong for funding his clinical assistant professorship.

Contributors GT: data extraction, analysis, manuscript draft and critical revision. MG: data analysis, manuscript critical revision. CI, KSL, YYT, IL, GB, GL, KPL, MD: data interpretation, critical revision of manuscript. TL, MCSW: conception of study, data interpretation, critical revision of manuscript.

Funding This study was funded by the Croucher Foundation of Hong Kong (10.13039/501100001692).

Competing interests None declared.

Provenance and peer review Not commissioned; externally peer reviewed. (c) Article author(s) (or their employer(s) unless otherwise stated in the text of the article) 2018. All rights reserved. No commercial use is permitted unless otherwise expressly granted.

\section{REFERENCES}

1 Carabello BA, Paulus WJ. Aortic stenosis. Lancet 2009;373:956-66.

2 Osnabrugge RL, Mylotte D, Head SJ, et al. Aortic stenosis in the elderly: disease prevalence and number of candidates for transcatheter aortic valve replacement: a meta-analysis and modeling study. J Am Coll Cardiol 2013;62:1002-12.

3 Mack MJ, Leon MB, Smith CR, et al. 5-year outcomes of transcatheter aortic valve replacement or surgical aortic valve replacement for high surgical risk patients with aortic stenosis (PARTNER 1): a randomised controlled trial. Lancet 2015;385:2477-84.

4 Anand A, Harley C, Visvanathan A, et al. The relationship between preoperative frailty and outcomes following transcatheter aortic valve implantation: a systematic review and meta-analysis. Eur Heart J Qual Care Clin Outcomes 2017;3:123-32.

5 Silaschi M, Conradi L, Seiffert M, et al. Predicting Risk in transcatheter aortic valve implantation: comparative analysis of EuroSCORE II and established risk stratification tools. Thorac Cardiovasc Surg 2015;63:472-8.

6 Vassiliou V, Chin C, Perperoglou A, et al. 93 Ejection fraction by cardiovascular magnetic resonance predicts adverse outcomes post aortic valve replacement. Heart 2014;100:A53.2-A54.

7 Weinberg EO, Shimpo M, Hurwitz S, et al. Identification of serum soluble ST2 receptor as a novel heart failure biomarker. Circulation 2003;107:721-6.

8 Dieplinger B, Egger M, Haltmayer $M$, et al. Increased soluble ST2 predicts longterm mortality in patients with stable coronary artery disease: results from the Ludwigshafen risk and cardiovascular health study. Clin Chem 2014;60:530-40.

$9 \mathrm{Yu}$ J, Oh PC, Kim M, et al. Improved early risk stratification of patients with STsegment elevation myocardial infarction undergoing primary percutaneous coronary intervention using a combination of serum soluble ST 2 and NT-proBNP. PLOS One 2017; 12:e0182829.

10 Jin M, Wei S, Gao R, et al. Predictors of long-term mortality in patients with acute heart failure. Int Heart J 2017;58:409-15.

11 Sinning C, Kempf T, Schwarzl M, et al. Biomarkers for characterization of heart failure - Distinction of heart failure with preserved and reduced ejection fraction. Int J Cardiol 2017;227:272-7.

12 Schmid J, Stojakovic T, Zweiker D, et al. ST2 predicts survival in patients undergoing transcatheter aortic valve implantation. Int J Cardiol 2017;244:87-92.

13 Stundl A, Lünstedt NS, Courtz F, et al. Soluble ST2 for risk stratification and the prediction of mortality in patients undergoing transcatheter aortic valve implantation. Am J Cardiol 2017;120:986-93.

14 Lindman BR, Breyley JG, Schilling JD, et al. Prognostic utility of novel biomarkers of cardiovascular stress in patients with aortic stenosis undergoing valve replacement. Heart 2015;101:1382-8.

15 Wernly B, Lichtenauer M, Jirak P, et al. Soluble ST2 predicts 1-year outcome in patients undergoing transcatheter aortic valve implantation. Eur J Clin Invest 2017;47:149-57.

16 Grantham JA, Borgeson DD, Burnett JC. BNP: pathophysiological and potential therapeutic roles in acute congestive heart failure. Am J Physiol 1997;272:R1077-83.

17 Griesenauer B, Paczesny S. The ST2/IL-33 Axis in immune cells during inflammatory diseases. Front Immunol 2017:8:475.

18 Yanagisawa K, Takagi T, Tsukamoto T, et al. Presence of a novel primary response gene $\mathrm{ST} 2 \mathrm{~L}$, encoding a product highly similar to the interleukin 1 receptor type 1. FEBS Lett 1993;318:83-7.

19 Demyanets S, Kaun C, Pentz R, et al. Components of the interleukin-33/ST2 system are differentially expressed and regulated in human cardiac cells and in cells of the cardiac vasculature. J Mol Cell Cardiol 2013;60:16-26.

20 Mildner M, Storka A, Lichtenauer M, et al. Primary sources and immunological prerequisites for SST2 secretion in humans. Cardiovasc Res 2010;87:769-77.

21 Luk KS, Ip C, Gong M, et al. A meta-analysis of soluble suppression of tumorigenicity 2 (sST2) and clinical outcomes in pulmonary hypertension. J Geriatr Cardiol 2018. 